\begin{tabular}{l|l} 
REVISTA Revista Educación \\
EDUGACIÓN & $\begin{array}{l}\text { ISSN: 0379-7082 } \\
\text { ISSN: 2215-2644 } \\
\text { revedu@ gmail.com } \\
\text { Universidad de Costa Rica } \\
\text { Costa Rica }\end{array}$
\end{tabular}

\title{
Modelo contextualizado de inclusión educativa
}

Aguinaga-Doig, Silvia; Rimari-Arias, Miguel; Velázquez-Tejeda, Míriam Encarnación

Modelo contextualizado de inclusión educativa

Revista Educación, vol. 42, núm. 2, 2018

Universidad de Costa Rica, Costa Rica

Disponible en: http://www.redalyc.org/articulo.oa?id=44055139007

DOI: https://doi.org/10.15517/revedu.v42i2.23885

Esta obra está bajo una Licencia Creative Commons Atribución-NoComercial-SinDerivar 3.0 Internacional. 


\title{
Modelo contextualizado de inclusión educativa
}

\author{
Contextualized model for educational inclusion
}

Silvia Aguinaga-Doig [1]

DOI: https://doi.org/10.15517/revedu.v42i2.23885

Institución Educativa Inicial No 004 "Angelitos de Maria”, Redalyc: http://www.redalyc.org/articulo.0a?id=44055139007 Perú

ginadoig@gmail.com

Miguel Rimari-Arias [2]

Universidad San Ignacio de Loyola Facultad Educación,

Escuela de Post Grado, Perú

mrimari@usil.edu.pe

Míriam Encarnación Velázquez-Tejeda [3]

Universidad San Ignacio de Loyola Instituto de

Investigación, desarrollo e Innovación, Perú

miriamv2002@yahoo.es

Recepción: 20 Abril 2016

Aprobación: 20 Marzo 2018

\section{Notas DE AUTOR}

[1] Licenciada en Educación Inicial por la Universidad Nacional Pedro Ruiz Gallo, Lambayeque, Perú y Magister en Didáctica de la Educación Inicial. Otorgado por la Universidad San Ignacio de Loyola, Lima, Perú. Es docente de la Institución Educativa Inicial N004 Angelitos de María, Chiclayo, Perú y docente de pregrado de la carrera de Educación Inicial, Primaria y Secundaria de la Facultad de Educación y en la Universidad Nacional Pedro Ruiz Gallo y de la Universitaria Católica, Santo Toribio de Mogrovejo en Chiclayo. Ha recibido varios cursos de postgrado como: Microsoft office specialist - Mos Master 2014-2015; Ofimática empresarial con certificación internacional-2014- 2015, Inglés, Nivel Avanzado -2015 y Diplomado en Tecnología de la Educación Inicial -2004. Ha participación en varios eventos científicos como ponente en la Pontificia Universidad Católica de Perú (PUCP) (2016), en la Universidad Federico Villarreal Lima (2015) y como Comentarista y Tallerista en el I Congreso Internacional de Educación Inicial en la Dirección de Educación de Lambayeque- 2011. Alcanzó el Primer Puesto para la beca otorgada por el Presidente de la República y el Ministerio de Educación para cursar la Maestría en Didáctica de la Educación Inicial en Lima, (2015), recibió la Orden al mérito Mujer lambayecana destacada en el desempeño de su profesión por el Gobierno Regional Lambayeque, (2013) y recibió una Resolución de la gerencia regional de educación 033-2013 por participar en el 1er. Concurso de Experiencias Científicas Inter Instituciones Educativas del Nivel Inicial- 2012.

[2] Bachiller en Educación por la Pontificia Universidad Católica del Perú; y Magister en Gestión de Instituciones Educativas otorgado por la Universidad Católica San José. Es docente universitario de pregrado de la facultad de Educación y de postgrado donde desarrolla la disciplina Metodología de la Investigación. Como coordinador de Investigación Educativa de la Escuela de Postgrado de Educación de la Universidad San Ignacio de Loyola de 2008- 2015 asesoró varias tesis de maestría con resultados satisfactorios. Ha escrito y publicado varios artículos pedagógicos en revistas pedagógicas de Perú, como SIGNOS, del Consorcio de Centros Educativos Católicos; en EDUCANDO de la Institución Docente Derrama Magisterial y en AUTOEDUCACIÓN del Instituto de Pedagogía Popular. Los temas abordados se han orientado fundamentar la gestión del docente y el aporte de modelos de documentos normativos (elaboración del proyecto educativo institucional, plan anual de trabajo, proyectos de innovación, entre otros), desarrollo y evaluación por competencias (elaboración del proyecto curricular de centro, elaboración de unidades didácticas, evaluación por competencias, los indicadores de evaluación) y habilidades blandas para los profesionales en educación (couching y empowermen educativo, autoestima docente, habilidades directivas, la asertividad en educación). Ha participado en varias investigaciones en los temas: El desarrollo y evaluación por competencias y el desarrollo de las habilidades blandas para los profesionales en educación (couching y empowermen educativo, autoestima docente, habilidades directivas, la asertividad en educación), cuyos resultados lo ha presentado en varios eventos en calidad de ponente en la Universidad Pontificia Universidad Católica de Lima y la Universidad San Ignacio de Loyola de 2008- 2015 asesoró varias tesis de maestría con resultados satisfactorios

[3] Doctora en Educación otorgado por: Universidad Potinficia Cathólica de la Florida, Estados Unidos, Magister en Psicología Educativa, por la UCP Enrique José Varona de la Habana y Licenciada en Educación. Se desempeña como Docente investigador de la Escuela de Postgrado de la Universidad San Ignacio de Loyola- Perú, donde desarrolla la docencia en postgrado y asesora tesis de maestría. Ha publicado varios aartículos científicos y dos libros: En la revista Páginas de Educación. Vol.9, Núm.; Revista Educación Vol. No 9 - Año VII (ISSN 1853-1318/ Argentina, 2017; Revista Iberoamericano de Educación REICE Vol. 15 No.2- 2017;. Revista de Educación de la UNCP, Huancayo, Perú, 2008. Libros: La comprensión de lectura. Fondo Editorial de la UCH. ISBN 978-612-4109-01-0, Perú. 2011 y Estrategias didácticas para enseñar y aprender la Lengua Española. Fondo Ed. Pedagógico, San Marcos Lima, 2003. Docente Universitaria de nacionalidad cubana: En Cuba se desempeñó como Docente, Decana de la Facultad de Educación y Vicerrectora Académica de la Universidad de Ciencias Pedagógicas Pepito Tey, de (1989 al 2009).En el Perú desde el 2010 ha sido Docente de Postgrado de la Universidad San Ignacio de Loyola, 2014- 2018, Universidad Antonio Ruiz de Montoya, 2016, Universidad Enrique Guzmán y Valle 2010- 2017, Universidad Peruana Global 2012- 2014, Universidad Peruana Unión de 2012- 2013. Ha participado en varias investigaciones y asesorado más de 50 tesis de maestría y doctorado; ha asistido a varios congresos Internacionales de Pedagogía: 2003, 2005, 2007; congreso Internacional de Lectura Habana 2007, 2008, 2010 y 2011 en Cuba, Perú y Brasil como ponente y jurado. 


\section{Resumen:}

La presente investigación sistematiza los referentes teóricos y metodológicos de la educación inclusiva y su incidencia en la formación integral de niñas y niños estudiantes de la educación inicial. Responde a un llamado del Ministerio de Educación de Perú dirigido a realizar propuestas para transformar la práctica del docente desde el programa de maestría cursado en la Universidad San Ignacio de Loyola. La metodología utilizada es cualitativa educacional de tipo aplicada, se vale de los procesos cualitativos y cuantitativos que ayudan a obtener la información, analizarla, interpretarla e identificar las categorías emergentes para proponer la transformación. Se emplean diferentes métodos, técnicas e instrumentos que como parte del diagnóstico de campo permitieron constatar el estado actual del fenómeno investigado en la práctica pedagógica. El estudio aborda los referentes teóricos acerca del enfoque de sistema, el socioformativo, el sociocultural y la didáctica general que le dan rigor científico a la propuesta. Como resultado se propone un modelo educativo que integra, desde la gestión y el liderazgo compartido, un sistema de estímulos conducidos por especialistas en permanente aprendizaje, que se concreta en acciones inclusivas orientadas al trabajo cooperativo y desarrollo temprano de las técnicas de intervención a favor del alumnado. Finalmente, se propicia el fortalecimiento de las habilidades diferentes al reorganizarse las aulas con mayor espacio que facilita la realización de las actividades pedagógicas más creativas y estimulantes.

Palabras Clave: Modelo de inclusión, habilidades diferentes, trabajo cooperativo, proceso de inclusión, gestión educativa.

\section{Abstract:}

The present research systematizes the methodological and theoretical referents in inclusive education and the incidence in the integral formation during the initial education ofPeruvian children. This study responds to the request of the Ministry of Public Education of Perú to write proposals to transform the practice in teachers pursuing the master's program atUniversidad San Ignacio de Loyola. An applied educational qualitative methodology was used. It consisted of qualitative and quantitative processes to obtain, analyze and interpret information as well identifying emergent categories to propose a transformation. Different methods, techniques and instruments used during the field diagnosis allowed to confirm the current situation of the investigated phenomenon in regards to teaching practices. The study under took the theoretical referents related to the system approach, socio educational aspects, socio cultural aspects and general didactics which contribute to the proposal scientific rigor. As a result, an educational model was proposed. This model integrated educational management and leadership shared with a of stimuli system conducted by specialists in continuous learning together with concrete inclusive actions oriented to cooperative work and the early development of intervention techniques to favor the students. Finally, different skills were reinforced through classroom organization with greater space for the realization of more creative and stimulating pedagogical activities.

KEYWORDS: Inclusion model, different skills, cooperative work, inclusion process, educational management.

\section{INTRODUCCIÓN}

La inclusión educativa es un derecho humano que ha emergido como un tema de interés mundial. Si bien fue implementada en el Perú desde el año 2003 al calor de la Declaración de Salamanca de 1990 y si bien en su aplicación en la práctica pedagógica se registran avances significativos, aún se aprecian discordancias e indiferencias que reclaman reflexiones al respecto. Esta realidad es paralela a la falta de interés de la sociedad por la aceptación de las personas diferentes con derechos humanos igualitarios.

Los enfoques actuales de la educación como proceso de desarrollo humano centran su atención en una escuela más formativa, de mayor apertura, que responda a la diversidad y cuyas estrategias de enseñanzaaprendizaje compensen las demandas cognitivas, afectivas y volitivas del alumnado para potenciar su desenvolvimiento sin discriminación de género, raza, cultura, credo, sexo o discapacidad. Todo ello desde un enfoque didáctico que potencie el aprender a ser, aprender a conocer, aprender a hacer y aprender a vivir en unidad como expresa Delors (1996).

Las investigaciones de Abu-Heran Abukhayran, Domingo y Pérez-García (2014), Cruz (2014) y García (2014) analizan, comparan y concluyen que la inclusión tiene desiguales ritmos de desarrollo y manera de presentarse en la práctica pedagógica. Si bien existen procesos incipientes de aplicación, también es innegable que, en las aulas, se alerta la presencia de acciones excluyentes que son distantes al objetivo rector. Al respecto, Molina (2010) describe en su tesis los problemas de inclusión social y el diseño de un modelo basado en la sensibilización de docentes de pregrado dentro de una institución educativa de Cuenca, Ecuador. Todo ello 
induce a promover propuestas transformadoras que se consoliden por su calidad en la práctica educativa en el marco de una mejora continua.

En el ámbito nacional, Choza (2010) y Solano (2010) en sus investigaciones exponen y critican la preparación y actitudes excluyentes de algunos grupos docentes en la práctica escolar. En su conclusión, destaca que la problemática existe, incluso, sin ser conscientes de esos modos de actuación que marginan al alumnado; insisten en la necesidad de implementar propuestas y modelos alternativos de educación que capaciten al personal para proyectar y llevar a cabo una educación inclusiva que en realidad responda a la diversidad y sea eficiente.

La investigadora ha observado en la práctica pedagógica que existen en los salones de clases comportamientos de indiferencia y rechazo hacia el alumnado con habilidades diferentes. Esto les provoca aislamiento respecto a sus pares y, en general, actitudes de inseguridad. Esas manifestaciones negativas que ocurren entre el mismo estudiantado son propias de la edad del grupo infantil, producto de no comprender por qué unos compañeros o unas compañeras son de una u otra manera. De ahí la relevancia de la acción profesional orientada por procesos educativos y didácticos sólidos, preventivos y oportunos.

En ese comportamiento de aversión por determinados grupos de estudiantes que presentan alguna diferencia en el desarrollo físico o psicológico se demuestran las incompetencias del personal docente ante el enfoque de inclusión social desde una pedagogía humanista que promueve el desarrollo integral del sujeto. Ello revela desconocimiento de la pedagogía especial y la falta de aplicación de métodos y estrategias psicopedagógicas inclusivas que potencian el desarrollo integral de la personalidad. Es imprescindible conducir el proceso de enseñanza-aprendizaje de manera que incida positivamente, en la formación de la personalidad de las personas menores. Esta situación es discordante, porque en los documentos normativos curriculares se precisa el trabajo de orientación inclusiva que debe hacer la escuela como institución democrática, tolerante, flexible y abierta a la inclusión social.

En la práctica pedagógica aparece una contradicción externa entre el nivel de educación inclusiva a lograrse en la institución educativa y las actitudes negativas del estudiantado, a la que se le suman las deficiencias que presentan los sujetos profesionales al dirigir el proceso de enseñanza-aprendizaje desprovisto del enfoque inclusivo. En tal sentido, el objetivo del artículo se orienta a revelar las causas que originan el problema y presentar un modelo contextualizado de inclusión para estudiantes de educación inicial con habilidades diferentes.

\section{REFLEXIONES ACERCA DE LOS MODELOS Y EL TRABAJO COOPERATIVO}

Con el paso de los años y el desarrollo de las ciencias, los modelos pedagógicos surgen con enfoques teóricos que inciden en los sistemas educativos en general. Cualquier modelo educativo que se diseñe en la actualidad debe ajustarse a las indicaciones emitidas por los organismos internacionales relacionados con la educación para todas las personas y sin exclusión. Significa aplicar una concepción educativa que centre su accionar en una formación más cualitativa y que responda a la pedagogía de la diversidad para favorecer el desarrollo integral de la población estudiantil.

Para enfrentar el reto de la educación inclusiva se necesitan docentes con nivel de profesionalismo alto; con compromisos en las ciencias de la educación; con sólidos recursos psicopedagógicos para aplicarlos en su desempeño y poder potenciar las capacidades, la comunicación asertiva, la socialización, el respeto, la tolerancia y las habilidades de estudiantes, cualquiera sea su condición, con la finalidad de contribuir a su desarrollo integral.

El enfoque de aprendizaje por competencias dentro del grupo de la alternativa socioformativa concibe la adquisición del conocimiento desde posiciones protagónicas de cada estudiante; privilegia los saberes previos del contexto, la problematización del contenido de forma interdisciplinaria con la propuestas de actividades que no estimulan el pensamiento, el lenguaje, la comunicación y la socialización en el estudiantado. En 
ese sentido, surge este enfoque de Tobón y Jaik (2012) con la perspectiva de mejorar la gestión curricular, la didáctica, la evaluación por medio de proyectos pertinentes, proyectos éticos de vida, aprendizajes colaborativos y metacognitivos que inciden en la formación integral del alumnado

El enfoque socioformativo ofrece los argumentos didácticos para encarar la enseñanza desde posiciones dialécticas y se caracteriza por los siguientes principios: pensar complejamente para ser mejores; proyectarse metas concretas; formarse mediante la colaboración y socialización; la formación íntegra del individuo depende de la sociedad; parte de la sencillez a lo complejo de la acción; los problemas son oportunidades para el desarrollo; la formación es emprendimiento; la mejora continua, y, por último, demostrar la actuación con evidencias. Diseñar y ejecutar la clase contemporánea desde estos principios le da lógica y ritmo a la función docente, coloca al alumnado como protagonista activo en el proceso de asimilación, procesamiento, comprensión, asunción de posturas y aplicación del conocimiento en las actividades de aprendizaje.

Desde estos referentes, la labor docente se orienta a la preparación constante en el estudio y empleo de métodos y estrategias de formación y desarrollo de habilidades y competencias para la vida en la sociedad. De esa manera, se redefinen los conceptos de equidad, justicia y bienestar para asumir posiciones activas, democráticas desde la flexibilidad que desencadene el potencial humano individual y el desarrollo integral en distintos contextos de actuación.

Según los fundamentos del enfoque sistémico, la estructura de elementos interrelacionados entre sí favorece la resolución de problemas y conlleva a una organización de orden secuencial que puede estar sujeta a cambios y mejoras. El enfoque de sistemas es conducido por la lógica hacia fines resolutivos, al crear un proceso cíclico que parte por identificar el problema, generar alternativas, elegir una de ellas, poner en práctica la seleccionada, determinar la eficiencia del proyecto y evaluar cada tiempo según las necesidades (van Gigch, 1987).

En ese orden, Senge (2000) sostiene que los sistemas son capaces de cambio y avance al desarrollo a través de las organizaciones y el aprendizaje. Es decir, para un individuo que quiere liderar procesos continúa siendo un reto producir efectos positivos, pero se sabe que estos no se consiguen de manera aislada, sino en la unidad y coherencia con el colectivo. Por ello, superar las complicaciones en una sociedad global como la peruana y alcanzar resultados positivos en la práctica implica un aprendizaje permanente y un liderazgo que en las instituciones educativas se debe afrontar; no solo con la instauración de un sistema de acciones para establecer soluciones, sino a través del diálogo, la comunicación y las interrelaciones permanentes en el trabajo, para que de conjunto orienten y apliquen acciones loables que remedien las situaciones de forma creativa. En conclusión, una estrategia interventiva basada en el conocimiento, la cooperación y la unidad de acción contribuiría al desarrollo del trabajo en equipo y el aprendizaje sería fruto de la socialización.

Desde estas posiciones, se necesita implementar enseñanzas que se orienten al desarrollo del aprendizaje cooperativo por su contribución, no solo a la esfera instructiva, sino por lo que aporta a las relaciones interpersonales, a la socialización, a la comunicación personal, a cómo aprenden unas personas de otras, cómo desarrollan la ayuda mutua, la solidaridad, el respeto, la tolerancia, el compañerismo y el sentido de partencia, entre otros beneficios, de acuerdo con el modelo Sociocultural de Vygotski, (1979). Se ha demostrado en las investigaciones que cuando el alumnado es bien orientado y guiado por profesionales competentes, se logra el tránsito progresivo de la zona de desarrollo actual a la próxima, al promover cambios notables en las formas de pensar, sentir y hacer del estudiantado, como refieren Castellanos et al. (2007).

A partir de estos rasgos se sostiene el valor de la puesta en práctica de proyectos innovadores en la resolución de problemas, como se plantea en los temas de inclusión desde todos los puntos de vista, de tal forma que la cooperación, la ayuda mutua, la socialización y el diálogo resultan un vehículo idóneo para la práctica de procesos inclusivos en las escuelas (Moriña, 2011). Así la estrategia cooperativa pretende que el alumnado realice acciones con objetivos comunes a partir del trabajo en pequeños grupos por las potencialidades que tiene este para fomentar el desarrollo de las capacidades, las actitudes como la responsabilidad, la solidaridad, la ayuda mutua y su contribución al desarrollo humano en su generalidad. 
En relación con lo anterior, salta a la vista el aprendizaje mediado porque permite desplegar el potencial cognitivo en las acciones al realizar la actividad, tanto en quien ofrece la ayuda como quien la recibe. Ello permite conjugar la intención de dar y recibir información, actitudes y procederes que orientan la actividad y los objetivos hacia donde, al regular el comportamiento, se requiere, de forma consciente, el interés por compartir, respetando la individualidad y tratando de hacer emerger lo nuevo, lo complejo y lo desafiante para que sirva de un incentivo a la motivación por el aprendizaje (Feuerstein, citado por Kholer, 2004).

Los estudios actuales aseveran que el aprendizaje es un proceso dialéctico, complejo, social e individual que en su asimilación, procesamiento y aplicación favorece la formación y el desarrollo de capacidades y habilidades en los infantes. Cuando este camino es conducido sabiamente con el compromiso docente, el nivel de progreso es alto, se presentarán modificaciones permanentes en el estudiantado y desarrollará tareas que aumentarán su complejidad de forma independiente, siempre que se privilegien los procesos interactivos y de socialización en su ejecución (Vygotski, 1979, citado por Ruiz y Estrevel, 2010).

$\mathrm{Al}$ analizar estos puntos de vistas y contrastarlos con la práctica educativa, se identifica, de forma persistente, la idea que relaciona las capacidades de estudiantes con la edad madurativa, sin valorar las diferencias en las capacidades, los ritmos y los estilos; estandarizan, de esta manera, el proceso y los resultados del aprendizaje. Sin embargo, el enfoque vygotskiano y de quienes je siguen sugieren la posibilidad de aprendizaje infinito de cada ser humano, siempre que se activen los estímulos externos e internos. Ello tiene que ver con la comunicación asertiva, la orientación y la calidad de la actividad de aprendizaje, sea cual fuere su condición de necesidad especial.

A propósito de las experiencias desarrolladas con la aplicación del enfoque socio cultural, GonzálezMoreno (2015) afirma que, mediante el método experimental que propone Vygotsky, es posible la formación de la función simbólica siempre que el trabajo docente oriente la realización de actividades lúdicas variadas que promuevan la creatividad, la interacción y asumiendo distintos roles dentro del juego temático. Desde luego, si en las aulas se potencia con mayor énfasis el juego como método y vía para motivar, estimular el interés, las capacidades y la recreación, los grupos de infantes sentirían más placer y alegría por lo que hacen. Desde esas perspectivas y organizando las aulas con recursos didácticos adaptados a los intereses de la niñez, se estimula y potencia la creatividad.

Un componente importante para llevar a cabo la redistribución de las aulas es la gestión educativa definida como el proceso de organización, planificación, ejecución, control y evaluación. Estos son lineamientos de la disciplina higiene escolar para sostener el proceso educativo mediante la administración de las instituciones como organizaciones sociales (Marín, 2006, citado por Torrealba, Soto y Piña, 2010) que debe tenerse muy en cuenta en todo proceso educativo.

\section{LA GESTIÓN INSTITUCIONAL ANTE LA INCLUSIÓN EDUCATIVA}

La Unesco (2011) plantea que el término gestión se puede definir desde cinco perspectivas: una centrada en la movilización de recursos; otra definida por la priorización de procesos; la interacción entre miembros; la comunicación y, finalmente, la que parte de los procesos que relacionan la gestión al aprendizaje. En consecuencia, se asumirá la conjunción de varias perspectivas, definiéndola como el paso constante de articular acciones, recursos, aprendizajes, estrategias, capacidades, estilos hacia un objetivo común, en donde es imprescindible la visión compartida, generación de valores, representaciones mentales e interacciones al interior de una comunidad educacional.

El enfoque estratégico de la gestión escolar toma como base la misión y visión de una institución y, a partir de ahí, dirige y planifica todas las acciones que deben conducir el desarrollo de la gestión escolar. Son acciones compartidas por personal directivo, personal docente, personal auxiliar, área de psicología, personal de servicios, madres, padres y estudiantes. Ante esa posición las estrategias deberán alinear actores y recursos en la misma dirección, con el fin de contribuir al desarrollo integral de sus infantes y que se refleja en los modos 
de actuación y en las transformaciones alcanzadas a corto, mediano y largo plazo (Alianza por la Calidad de la Educación, 2010a, b).

Ello tiene que ver con el nivel de desarrollo del liderazgo educacional, entendido como la capacidad que posee la alta dirección escolar orientada a mejorar el desempeño de la institución educativa, a través de la creación de un clima organizativo de socialización, tolerancia y respeto, con el fin de potenciar el desarrollo del aprendizaje integral según las capacidades, estimular el esfuerzo y la efectividad del equipo docente y administrativo como aseveran Pedraja-Rejas, Rodríguez-Ponce y Villarroel-González (2012).

En Irlanda (McGlynn y London 2013) se sugiere que el liderazgo podría impactar a favor de la inclusión, si se compartiera el significado de esta ideología e institucionaliza como práctica la colaboración. Igualmente en Australia (Bristol, 2015) se reflexiona acerca de la actuación de maestras y maestros como líderes del proceso educativo y como un ente dinámico, pues, que a partir de su intervención, se puede innovar la enseñanzaaprendizaje, reforzando la idea que más allá de las políticas está el fomento de la cultura inclusiva desde el nivel inicial, tal lo afirman Novachevska, Dimchevska, Pavlovska, Chichevska-Jovanova y Dimitrova-Radojichikj (2011).

Concerniente a la organización de los equipos de trabajo, es preciso reconocer que el profesorado ejerce una influencia preponderante en la formación de la personalidad de alumnas y alumnos, aunque es real que también reciben las influencias educativas de la familia y de otros agentes en los diversos contextos de actuación. Sin embargo, los conocimientos, las habilidades, la disposición para hacer, el interés y las motivaciones traspasan los límites de la gestión, incluso cuando no existe una adecuada implementación de infraestructura y equipamiento. Es necesario reforzar la evaluación del desempeño y el reconocimiento mediante la asignación de incentivos que evitarían la movilización docente en la búsqueda de mejores espacios laborales que cubran sus demandas, tanto económicas, sociales, de rendimiento, motivación y compromiso (Pedraja-Rejas et al., 2012).

Las actividades de inclusión educativa constituyen un desafío para quienes son profesionales de la educación, porque en su generalidad no disponen de preparación, no han recibido la capacitación para enfrentar la tarea y en ocasiones se sienten impotentes ante las demandas del organismo y las familias. En tal sentido, el personal directivo enfrenta posturas inadecuadas para que maestras y maestros puedan responder con eficiencia al tratamiento de los objetivos y las tareas de inclusión social en las aulas.

$\mathrm{Al}$ respecto, existe un grupo de docentes con capacidad e interés que se autoprepara por iniciativa y responsabilidad ante la profesión, otros con capacidad, pero escaso compromiso de superación y muestra de desconocimiento ante el tratamiento a seguir y un tercer grupo que está desmotivado, con signos de agotamiento y no sabe cómo proceder. De esta manera las tareas de inclusión educativa constituyen un desafío que hay que enfrentar con una adecuada capacitación didáctica que avale al colectivo pedagógico para transformar la realidad con eficiencia para no comprometer el futuro del alumnado.

Cejas (2009) considera importante la motivación y los estímulos docentes para que su desempeño en esta esfera sea eficaz e impere un clima institucional de cordialidad, respeto, tolerancia y ayuda mutua al ofrecer las oportunidades para que todo el estudiantado desarrolle sus capacidades hasta donde le es posible. Es imprescindible considerar y vivenciar un ambiente de crecimiento desde las situaciones cotidianas de forma que permita poner en práctica su potencial cognitivo, afectivo y revelarlas a través de las actitudes, las creencias, los valores, sus motivaciones y establezcan relaciones cívicas y ciudadanas para la convivencia en democracia y armonía.

En resumen, la categoría gestión bajo el enfoque del desarrollo humano escolar reorienta el liderazgo basado en el diagnóstico, la comunicación, la socialización y la acción compartida. Ello demanda del ejercicio de sistemas de una relación dinámica, eficiente entre los miembros de los equipos y de un clima institucional que integre al colectivo en aras de cumplir las metas a corto, mediano y largo plazo para lograr un crecimiento cualitativo superior en la institucional. 


\section{El trabajo COOPERATIVo EN LAS AULAS: Retos y PERSPECTIVAS}

El trabajo cooperativo es una vía esencial para potenciar el desarrollo de las habilidades diferentes. Ello promueve la interacción comunicativa, la crítica, la valoración y la reflexión en las actividades de aprendizaje donde unos grupos o individuos imitan a otros en espacios dialógicos y de socialización. Qu (2011), en su estudio, muestra cómo los grupos de preescolares pueden orientar su ayuda al otro y de esta manera logran controlar también su propia actividad cuando al jugar comparten un objetivo común. Ello es un argumento natural del sentido social útil que posee y la escuela es el centro responsabilizado con la aplicación de estrategias cooperativas, socializadoras, lúdicas, creativas e inclusivas desde la atención individual y colectiva que demandan en su esfera cognitivo, afectivo, emocional y procedimental para contribuir al desarrollo integral del estudiantado.

Distintos estudios han demostrado que el trabajo cooperativo refuerza el desarrollo de las capacidades sociales y afectivo-volitivas, porque inciden en el desarrollo de la personalidad, la que se va moldeando según las necesidades que demuestran los niñps y las niñas en las actividades interactivas. La propuesta precisa con fuerza la necesidad de este trabajo desde las primeras etapas, indiscutiblemente, formadoras de la unidad integral que constituye la persona participante en procesos educativos.

Otro elemento fundamental está dirigido al fortalecimiento de las habilidades diferentes. A propósito, la UNESCO reconoce que cada ser es una individualidad con necesidades, intereses, capacidades y habilidades diferentes. Enfatiza que el concepto de inclusión educativa estuvo por mucho tiempo relacionado con personas con necesidades especiales; concepto tradicional que ha evolucionado y que hoy se acuña para referirse a las necesidades de aprendizaje de todas las personas (Opertti, 2005).

Se asume el enfoque integrador con equidad (Klingler y Vadillo, 2000), la teoría de Wallon, la plasticidad neuronal a la luz de la neuropsicología de Luria y la modificabilidad cognitiva de Feuerstein, así como el modelo de inclusión social (Palacios, 2008) y el enfoque de sistemas en el modelo propuesto que se caracteriza por involucrar a los agentes educativos que conforman una unidad de cooperación, donde cada aspecto de la gestión y la práctica pedagógica valora la posibilidad de recibir el tratamiento que necesita, según las carencias y las fortalezas de manera individual y grupal.

La atención profesional a las necesidades de estudiantes de las escuelas precisa de la aplicación de procedimientos y pruebas psicopedagógicas para diagnosticar el nivel real de desarrollo de cada estudiante con el fin de caracterizarles y trazar el tratamiento a seguir desde una concepción de la inclusión integradora, que permita transitar a niveles de desarrollo superiores. Estos procedimientos de carácter interdisciplinario tienen como fin conocer no solo las debilidades, sino las fortalezas para orientar la labor educativa, así como contribuir a la preparación de la familia y la comunidad en general. Para que la institución sea profunda y reveladora del perfil del niño y de la niña deberá diseñar los planes interventivos y las modificaciones curriculares con un carácter personológico, donde se precise en qué áreas necesitan niveles de ayuda y el tipo de soporte que requieren. También es importante identificar el estilo y los ritmos de aprendizaje, cómo potenciar la motivación, su desempeño social, conocer las condiciones familiares, sociales, las deficiencias, cómo es el entorno escolar y constatar de qué manera el grupo se convierte en una fortaleza para el logro de los objetivos (Alianza por la Calidad de la Educación, 2010a, b).

La atención temprana es un componente por tratar de forma permanente en la etapa preescolar porque favorece su desarrollo. Al respecto, López (2012) refiere que esta actividad permite llevar a la práctica un conjunto de acciones orientadas a generar respuestas ante la presencia de las necesidades pasajeras o permanentes, y lo necesario que resulta conocer las influencias positivas del ambiente sociocultural y afectivo para lograr el equilibrio emocional y la formación integral de la niñez para la vida.

Los aportes de la neuropsicología y la modificabilidad cognitiva dan cuenta de la importancia de fortalecer la comunicación asertiva, la esfera emocional y afectiva del alumnado al realizar cualquier actividad escolar. $\mathrm{Al}$ respecto, existen técnicas como la psicomotricidad, los juegos de roles y libres, la narración de cuentos, 
la música, la danza, la observación de la naturaleza y la expresión plástica que ponderan la proactividad individual. Su implementación debe considerar los ambientes, los horarios docentes y de recreación, con el propósito de cumplir los objetivos de intervención. En línea con ello Wallon realizó un estudio valioso acerca del desarrollo madurativo de infantes, basado en el aspecto psicomotor y social, que dio pie a que otras investigaciones declaran la relación entre la psicomotricidad y el aprendizaje (Martín, 2012).

La teoría sociocultural vygotskiana reconoce que el desarrollo es el resultado de un proceso de apropiación de la cultura, de la socialización, de vivencias y de experiencias que el medio proporciona al sujeto, que le sirven de herramientas en su desarrollo evolutivo y de reafirmación de sí mismo por medio de la imitación, la interacción y la asunción de roles. Al respecto, el psicólogo francés Wallon (2007) refiere que uno de los factores para explicar la evolución psicológica es el otro ser. Basa su teoría en la estrecha relación entre la madurez biológica y su relación con el medio social, la cual permite la observación, asimilación, comprensión y reorganización de todas las estructuras cognitivas. Por su parte, Ramenzoni, Riley, Shockley y Baker (2012) expresan los sustentos o requisitos que deben reunirse para la ejecución de tareas en grupos en las que predomina la relación interpersonal ante el rendimiento individual, de tal forma que aumenta en estabilidad, rendimiento y la actuación conjunta.

\section{Procedimientos metodológicos}

La metodología empleada durante el proceso investigativo fue la cualitativa educacional de tipo aplicada. En su desarrollo se integran los métodos cualitativos y cuantitativos que permiten obtener la información, analizarla, interpretarla e identificar las categorías apriorísticas y emergentes, contrastarlas y llegar a conclusiones aproximativas. Este tipo de investigación se caracteriza por sistematizar la teoría y relacionarla con la realidad educativa en la búsqueda de la objetividad y la producción del conocimiento para aplicarlo a través de propuestas, metodologías, estrategias y modelos educativos que contribuyan al desarrollo, la creatividad e innovar la práctica pedagógica (Cívicos y Hernández, 2007, citado por Vargas, 2009).

La base ha sido la experiencia profesional pedagógica en las aulas y del análisis de los documentos normativos que rigen la política educacional del enfoque de la educación inclusiva en la educación preescolar para contribuir a la formación integral de niñas y niños. Sin embargo, se aprecia una contradicción con la realidad que se concreta en el aislamiento y la incomunicación entre estudiantes en la práctica escolar y en relación con el nivel de gestión docente para aplicar una educación inclusiva de calidad.

\section{Diagnóstico}

El proceso de diagnóstico de campo se llevó a cabo en la institución educativa seleccionada, de manera intencional y criterial, por reunir las condiciones reales para cumplir el objetivo trazado por la investigación. Antes de iniciar la aplicación de los instrumentos se coordinó con personal directivo, docentes y alumnado de la escuela para explicarles los objetivos del trabajo y lo útil de su prestancia. Se previó una muestra intencionada, conformada por la directora, diez docentes, nueve auxiliares de educación, ocho madres de familia y 90 estudiantes. Se usaron técnicas de entrevista semi estructurada, videos, cuestionarios, guías de observación, fotografías, videograbaciones y material escrito según el tipo de instrumento.

\section{Proceso de categorización}

Los resultados se organizaron mediante el proceso de triangulación (Okuda y Gómez-Restrepo, 2005) en el que se analiza, interpreta y contrasta de forma integradora la información acopiada para arribar a las categorías emergentes que se confirman a partir de los hallazgos procesados por codificación cualitativa (Gibbs, 2012). 
En un primer momento se hizo la transcripción, codificación y categorización de los datos; en el segundo momento, la comparación, relación y clasificación de las categorías y en tercer momento: la triangulación, integración, interpretación y análisis de todas las técnicas y los instrumentos utilizados para llegar a las conclusiones relacionadas con el fenómeno estudiado. El proceso de categorización en cuanto a los principales hallazgos se resume como sigue:

\section{Categoría emergente 1: Gestión del proceso inclusivo}

En la institución educativa se aprecia como potencialidad la dotación de los recursos materiales esenciales para sus funciones y la constitución de un claustro pedagógico completo con disposición para llevar adelante la labor educativa; no obstante el proceso inclusivo que llevan a la práctica aparece en contra posición a la opinión del personal docente, administrativo, auxiliares pedagógicas, madre y padres de familia que no admiten la realidad y, por el contrario, demuestran estar conformes con su comportamiento. Sin embargo, las manifestaciones identificadas en las actividades técnico-pedagógicas confirman el inadecuado manejo de las estrategias que aplican al realizar la matrícula del alumnado.

\section{Categoría emergente 2: Manejo de estrategias para planificar, ejecutar y evaluar el proceso de aprendizaje de estudiantes con habilidades diferentes}

Los grupos de docentes aplican distintas estrategias para dirigir y evaluar el proceso aprendizaje de niñas y niños y tienen control de ello, aunque reflejan desconocimiento de los enfoques teóricos actuales para la educación inclusiva en relación con los métodos, los procedimientos, las estrategias y el apoyo especializado para llevar a cabo un trabajo didáctico coherente que incida en el desarrollo integral de los infantes. El personal directivo considera que el rol docente es importante, sin embargo, no existe un adecuado sistema de estímulos y reconocimiento que impulse sus compromisos en la práctica cotidiana; se observan niveles de estrés y resignación debido a las dificultades existentes desde el inicio del proceso de matrícula cuando las madres o los padres no reconocen que sus hijos o hijas son diferentes y cuando tienen que recibir al alumnado en sus aulas sin sentirse con la preparación para conducir el proceso educativo. En sentido general, el equipo pedagógico necesita de un trabajo cohesionado y alcanzar un nivel de profesionalismo superior, para dar un tratamiento didáctico al proceso de enseñanza- aprendizaje.

\section{Categoría emergente 3: Actitudes de estudiantes}

La observación prevista reveló que niñas y niños en general se comunican, comparten y asumen sus responsabilidades, aunque con bajos niveles de aceptación de niñas y niños con necesidades especiales, reflejado en actos de indiferencia, de agresividad de palabras o acciones y actitudes discriminatorias entre infantes, madres y padres. La interacción entre el alumnado en la realización de las actividades y en el grupo en general es pobre y, como consecuencia, reportan exclusiones como resultado de las actitudes de rechazo que influye en un comportamiento de inseguridad, aislamiento y dependencia a la auxiliar pedagógica, lo que denota falta de un tratamiento educativo adecuado, y de control docente y de la institución.

En conclusión, se constató que los procesos de inclusión practicados en la escuela son asistemáticos, a ello contribuye la falta de preparación profesional, la falta de gestión inclusiva y de didácticas. La responsabilidad de los procesos está a cargo de la directora, el equipo docente, las madres y los padres de familia que deben cohesionar las fuerzas para lograr el buen funcionamiento del colectivo en aras del desarrollo multilateral y armónico de la población infantil. 


\section{ANÁLISIS Y DISCUSIÓN DE LOS RESULTADOS}

Como resultado del proceso de reflexión se confirma, desde diversas fuentes, que el problema analizado radica en la estructuración de la gestión del proceso inclusivo a nivel interno. Tal como afirma la Unesco (2011), la gestión puede tener diferentes perspectivas como es la priorización de los procesos y la centrada en la interacción de los miembros de una comunidad escolar. Quiere decir que si la inclusión se convirtiese en el objetivo priorizado de una institución educativa todo el sistema se adaptaría sin inconvenientes, siempre que haya liderazgos con poder de convencimiento y visión compartida.

El diagnóstico presenta indicadores de un sistema inclusivo débil, dado que prescinde de claridad y concreción. Esto ocurre cuando al momento de la matrícula no se cuenta con el personal capacitado para realizar las evaluaciones psicopedagógicas. $\mathrm{Al}$ respecto, se precisa conocer la importancia de poner en marcha procesos integradores con el enfoque de sistemas (Senge, 2000), que no solo prevén la organización abierta al aprendizaje, sino cómo interactúan las partes al interior para conducir de manera lógica y cíclica la resolución de problemas existentes.

Los hallazgos en cuanto al desempeño docente en las aulas donde hay estudiantes con habilidades diferentes indican la necesidad de capacitación en los enfoques contemporáneos, fortalecer el nivel de profesionalismo, la autoestima profesional de manera que se aprecie un progreso en liderazgo educacional y, de esa forma, el clima institucional. Al respecto, Fernández y Hernández (2013) y Pedraja-Rejas et al. (2012) plantean que el liderazgo y la gestión del factor humano influyen de acuerdo con el contexto en la calidad del producto enseñanza- aprendizaje.

En la práctica pedagógica existen vacíos en cuanto a la atención pedagógica según las necesidades e intereses de infantes que se postergan, apartan y cuyo desarrollo integral, así, se limita. Frente a ello, resultan oportunas las técnicas de intervención sugeridas por Bernaldo (2012) que contribuyen a la atención temprana con un carácter preventivo y poseen argumentos teóricos de acuerdo con la edad del alumnado.

Los resultados muestran actitudes de indiferencia y agresividad entre estudiantes por la falta de sensibilización y trabajo educativo de grupo en las acciones institucionales. En este contexto existen pocos espacios para promover el desarrollo de las capacidades y las competencias del alumnado de manera que contribuya a la preparación para la vida. Esto se evidencia en las actitudes, en los modos de actuación de servicio para sí y para las demás personas (Tobón y Jaik, 2012).

Los soportes teóricos y metodológicos para atender los problemas de conducta y realizar trabajo cooperativo indican que es necesaria la organización del alumnado en pequeños grupos, asignándoles responsabilidades y proporcionándoles la oportunidad de ser partícipes activos en la realización de actividades orientadas a promover la creatividad en ambientes de trabajo que les estimulen a participar libremente y que incidan en su formación. Así, el beneficio no se limitaría a cierto grupo de estudiantes, sino que será una estrategia para contribuir al desarrollo de las capacidades de la diversidad del alumnado. Desde estas perspectivas se requiere fomentar un entorno escolar comunicativo enriquecedor que lo aleje de lo ordinario, según la experiencia de McAllister y Hadjri (2013).

Las madres y padres de familia no cuentan con herramientas para afrontar los efectos de las diferencias de hijos e hijas y, en ocasiones, se perciben períodos de duelo en las familias ante la noticia de recibir una persona especial, luego comienza a identificarse con la situación real que deben asumir y, por último, pueden tomar una de las siguientes decisiones: se vuelven conformistas ante la falta de recursos, motivaciones y conocimiento para superar las barreras o son conscientes del tratamiento afectivo y de atención para el desarrollo las habilidades diferentes y proporcionan toda clase de experiencia como paseos, juegos, terapias, educación, entre otras.

La problemática presentada se extiende en la práctica docente inclusiva que resulta un tema desafiante para la mayoría de estas docentes, lo cual les genera contradicciones para asumir su desempeño con el nivel de motivación y el compromiso ético que se necesita para ofrecer la orientación educativa en función de las 
características de los grupos de infantes que atienden, de manera que puedan ejercer una estimulación integral para la vida social.

\section{Propuesta del modelo de educación inclusiva}

En principio, el modelo de gestión educativa propuesto tiene el propósito de valorar e incluir de forma activa, en el sistema educativo, al alumnado con habilidades diferentes con el objetivo de orientar todo su potencial cognitivo, afectivo y volitivo en la actividad educativa, a través de la puesta en práctica de un plan permanente de acciones de carácter táctico y estratégico. Permite la reestructuración de los documentos de gestión (proyecto educativo institucional, plan anual de trabajo y proyecto curricular institucional), del programa de fortalecimiento de la autoestima y desarrollo interpersonal de los grupos profesionales; de la capacitación para todo el personal, las madres, los padres y el alumnado; y del mejoramiento de la infraestructura, con la propuesta de la redistribución de las aulas con la intención de aplicar las técnicas de intervención en locales especializados.

La propuesta se ha conformado desde las perspectivas del trabajo colectivo, promoviendo la activa participación y dinámica de la comunidad escolar tanto en la etapa inicial, en la ejecución, como en los resultados. Para lograrlo, se ponen en práctica distintos métodos, estrategias, metodologías, técnicas, instrumentos que permitan su aplicación y evaluación permanente de las actividades que contempla la alternativa establecida para lograr el trabajo cooperativo (Figura 1).

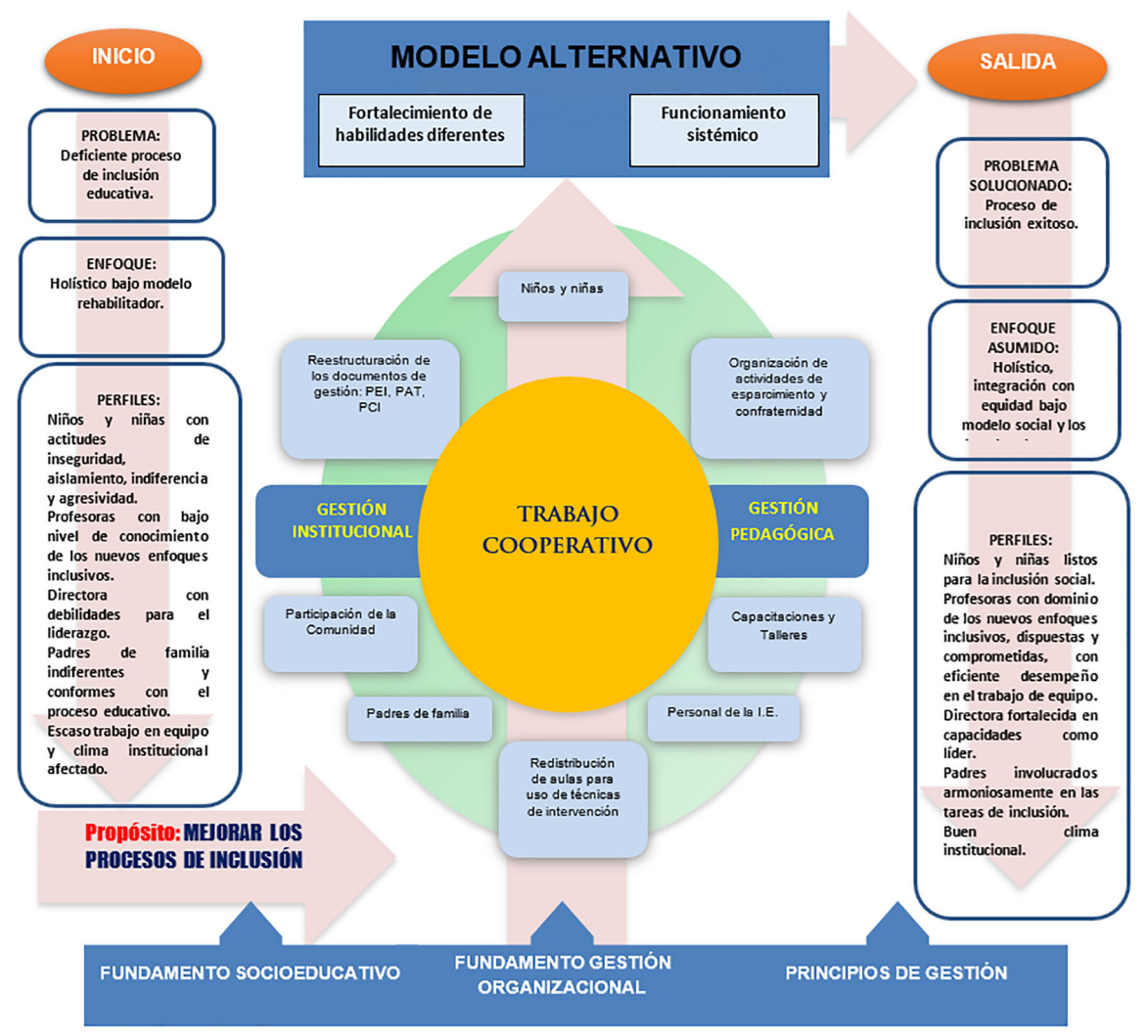

FIGURA 1

Estructura del modelo, refleja la propuesta, basado en el enfoque sistémico de van Gigch (1987).

Con la finalidad de cumplir los objetivos de las técnicas de intervención, se propone, ante todo, crear espacios innovadores que contribuyan a organizar un contexto favorable según el modelo propuesto. Esto implica redistribuir los ambientes para hacer uso óptimo de los medios materiales, recursos con que cuenta 
la institución e incluso se puede vincular a madres y a padres de familia a las acciones del juego educativo. La innovación de aulas sugiere cambios, no siempre asociados a la inversión material, económica, pero sí a la producción creativa, manual y práctica. Por ejemplo, hacer uso de material recuperable y adaptar los recursos existentes de manera que se considere la participación del alumnado organizado en los grupos de aulas predeterminadas por edades.

El juego infantil es una construcción social y cultural a través de la cual los niños y las niñas recrean las relaciones personales y sociales del entorno en el que viven y, así, se van apropiando del conocimiento y las habilidades para desempeñarse de forma gradual, según Vygotsky (1979, citado por MoragónAlcañiz y Martínez-Bello, 2016). Por ello se hizo necesario redistribuir las aulas para optimizar el tiempo dedicado al juego como actividad de aprendizaje esencial, los espacios y los recursos existentes. Las aulas se organizaron por especialidad como: psicomotricidad, minicocina, expresión plástica, construcción, teatro, música, investigación y tecnología. El estudiantado infantil rotaría por ellas para desarrollar el juego libre y de roles, la psicomotricidad y otras técnicas de intervención en espacios específicos como refiere Berges y Bounes (1983, citado por Bernaldo, 2012).

El modelo basado en el trabajo cooperativo y la redistribución de las aulas en grandes sectores para desarrollar el juego-trabajo incluye a todo tipo de estudiantes con la intención de promover el diálogo, la expresión democrática, la interacción, la cooperación en el trabajo en grupo, la valoración de las actividades realizadas, el saber explicar qué han hecho, cómo lo han hecho, para qué y cómo lo evalúan, de manera que se incentiven el intercambio, la ayuda mutua, las habilidades comunicativas y el aprendizaje compartido.

Antes de mencionar algunos ejemplos en cuanto al trabajo cooperativo a realizar en las aulas, resulta fundamental la realización de las evaluaciones psicopedagógicas como primera acción diagnóstica que permite conocer fortalezas y carencias para trazar la intervención a seguir de manera personalizada y también colectiva. Para ello, es imprescindible la incorporación de un equipo interdisciplinario de especialistas permanente en la institución. Sin embargo, no solo se precisa de la evaluación inicial sino también valorar el proceso que permita conocer para actuar de forma preventiva con toda la población infantil de 0 y 6 años. Ello formará parte del programa de atención temprana que incluye la aplicación de distintas técnicas de intervención con determinado objetivo para conocer con certeza la realidad de cada estudiante; entre las que se pueden emplear está: el cuento vivenciado, la danza terapia, la relajación y la expresión plástica.

El cuento vivenciado permite, mediante el lenguaje articulado, los movimientos y la gestualidad estimular las capacidades de escuchar, expresar, danzar, dialogar, interactuar y comunicar de manera integral. Es una actividad que favorece la comprensión, la imaginación y la fantasía, y experimentar corporalmente la secuencia de una historia escuchada, las habilidades intelectuales y las destrezas psicomotoras. Antes de su realización se deberán seleccionar los textos por temáticas, tipo de mensaje, gustos, preferencias y que respondas a los intereses, las necesidades comunicativas democráticas, a tal punto que puedan escoger el personaje con el que se identifican y establecer lazos afectivos con la persona adulta que lee o narra y, para lograr más satisfacción, se podría contar con la participación de padres y madres de familia.

La danza, por su naturaleza, influye en la sensibilidad humana. En la propuesta se emplea como una estrategia de enseñanza con un poder educativo, reeducativo y terapéutico de incalculable valor. Los estudios de la neuroeducación revelan que el uso de la danza y el baile como terapia incrementan las aptitudes físicas y motoras; mejoran el autoconcepto, la autoafirmación, la espontaneidad, la alegría y el entusiasmo; y potencian la proactividad del estudiantado al favorecer los fomentos de la independencia e integración social.

Una de las técnicas que más contribuye a la satisfacción y la disposición de niñas y niños es la relajación que, en su forma más común, debe ser practicada permanentemente en las aulas después de la realización de actividades que han requerido altos niveles de concentración. También se puede aplicar después de una sesión de psicomotricidad o podría incorporarse dentro de la propuesta con espacios de tiempo más largos y con la conducción de especialistas para que la relajación terapéutica tenga su lugar adecuado. Según Berges y Bounes (1983 citado por Bernaldo et al., 2012), la relajación terapéutica es un método que surge a partir de 
las tesis de Schultz y Ajuriaguerra (citados en Bernaldo, 2012), orientado a la reeducación de personas a través de tres fases que resultan ideales para infantes que manifiestan actitudes negativas para sus compañeros.

La expresión plástica no debe dejar de ser uno de los medios más usados en las aulas de preescolar, pues su carácter revelador del mundo interior, cómo comprende el infante su entorno, cuáles son los conocimientos y sentimientos que poseen cobran fuerza para definirla como un instrumento de evaluación, de seguimiento y como técnica de intervención. Como evaluación, valora el nivel de desarrollo actual, anticipando y previendo dificultades para el futuro. Como elemento de seguimiento, verifica la evolución al comparar sus trabajos que como técnica de intervención permite poner en práctica objetivos y contenidos a través del dibujo, la pintura, el collage y el modelado, entre otros.

La estrategia de trabajo cooperativo no solo es una propuesta para orientar el trabajo docente y contribuir con la dirección del aprendizaje y la socialización infantil, sino que orienta las acciones y se convierte en una guía para el desempeño pedagógico de la comunidad educativa. Así, el buen desempeño de los trabajos en equipo de manera cooperativa deberá realizarse con entusiasmo y con una actitud profesional positiva frente a los nuevos retos.

El desempeño docente se puede fortalecer con la realización de un programa de capacitaciones y de talleres de carácter teórico-práctico que aborden los enfoques inclusivos y que influirá en la preparación del equipo para orientar el proceso formativo inclusivo, así como implementar actividades de esparcimiento y recreación del personal docente como: visita a un centro de belleza, una noche de karaoke, visitas a museos, práctica de ejercicios físicos, observación de la naturaleza, entre otras, con el propósito de fortalecer la autoestima e integración del equipo. Su puesta en práctica permitirá fortalecer la autoestima profesional, el estímulo al reconocimiento y la promoción a los méritos laborales en estrecha relación con la calidad de los logros.

Este aspecto comprende, además, la gestión que involucra la responsabilidad social de las entidades públicas o privadas de orden educativo o no, que adoptan políticas participativas como puede ser: auspicio a las capacitaciones, actividades y eventos con la participación de sus profesionales especialistas y como parte observadora externa que contribuya al proceso de evaluación durante la etapa ejecutora de la propuesta.

Finalmente, es preciso reflexionar sobre el impacto que tendrá la puesta en práctica del modelo contextualizado inclusivo no solo en la comunidad educativa, sino en la comunidad en general. Al respecto, se constata que, con el trabajo sistemático de dos o tres docentes con un profesionalismo y vocación de servicio, la educación del alumnado y la orientación a madres y padres va a marcar la diferencia cualitativa y toda la institución educativa va a alcanzar el nivel deseado.

\section{Valoración de las potencialidades del modelo según consulta a especialistas}

El propósito de la valoración se orientó a determinar la eficacia del modelo basado en el trabajo cooperativo para mejorar procesos de inclusión educativa. Las personas participantes en esta fase poseen la autoridad para avalar la propuesta al contar con grados académicos y experiencia profesional reconocida. Este proceso se llevó a cabo con la participación de la magistra Vivian Maribel Villegas Ramos, licenciada en la educación inicial con estudios de docencia, gestión educativa y problemas de aprendizaje; cuenta con veintitrés años de servicio como maestra inclusiva y directora, y con la magistra Edith Marianela Luquillas Rodríguez, especialista en psicología educativa con veinte años de servicio y cuenta en su haber con la realización de varias investigaciones sobre inclusión social.

El promedio de los resultados de esta evaluación fue 99 puntos. Las valoraciones cualitativas califican la propuesta como muy buena, pues cumple con el propósito de mejorar los procesos de inclusión educativa. Asimismo, se concluye que el resultado es aplicable en otras instituciones educativas similares, siempre que se tengan en cuenta las características psicopedagógicas, sociales y culturales de los contextos donde se pretende aplicar. 


\section{Conclusiones}

La existencia de modelos educativos contextualizados con objetivos inclusivos es una exigencia de la inclusión educativa como parte del desarrollo humano. La gestión educativa debe integrar a toda la comunidad escolar y comprometerla en el fortalecimiento de una educación para el desarrollo humano. En tal sentido, se realizó un diagnóstico cualitativo en la institución educativa participante y como resultado del proceso investigativo se aporta, a la práctica pedagógica, un modelo contextualizado de inclusión educativa.

Si bien es cierto que en los primeros hallazgos de los resultados se constataron problemas identificados en tres aspectos: la gestión educativa, la participación docente y la conducta de los grupos de estudiantes; también es cierto que se identificaron fortalezas como la existencia de un claustro pedagógico formado para la labor escolar que trabaja con sentido de pertenencia, la alegría y la disposición de las niñas y los niños por aprender, el interés de las madres y los padres de familia por involucrarse y ayudar en las tareas de la escuela y, en general, la disposición del colectivo por avanzar y alcanzar los objetivos que impulsan su desarrollo del alumnado.

El proceso investigativo, en su generalidad, permitió arribar a las siguientes conclusiones como una síntesis de las categorías apriorísticas y emergentes acerca de la realidad educativa y de su posible transformación con el modelo educativo contextualizado diseñado como producto de carácter teórico - práctico:

Gestión del proceso inclusivo. Frente a las condiciones de los procesos de gestión en los centros de educación básica especial, la forma como se hace la distribución de estudiantes en las aulas y las características de la evaluación, resulta oportuno promover una gestión que valore la inclusión, que considere la incorporación de personal especializado para la evaluación psicopedagógica del alumnado, que le acompañe desde el inicio del ciclo escolar y que tenga en cuenta la nueva redistribución creativa de las aulas para organizar el seguimiento y la evaluación diferenciada.

Esto es posible si se logra una rigurosa gestión bajo los enfoques del factor humano y estratégico escolar para promover cambios significativos en la escuela, así como realizar convenios con instituciones públicas y privadas que provean de personal especializado como parte de un equipo de trabajo permanente.

Si se lleva a cabo una gestión para garantizar el liderazgo en la educación inclusiva, tal como se registra en documentos oficiales, como el proyecto educativo institucional, el plan anual de trabajo, el proyecto curricular del centro y el reglamento interno, se darán cuenta de que se trata de una política educativa y se tendrán que alinear de una manera u otra a los objetivos estratégicos de la escuela inclusiva.

Participación docente. El desconocimiento de enfoques inclusivos, el escaso manejo de estrategias pedagógicas y el poco compromiso con la mejora de la calidad de la enseñanza tienen consecuencias dentro de la educación inclusiva, tanto entre docentes, auxiliares de educación, madres y padres de familia y, en especial, con respecto a niñas y niños. Asimismo, la edad y el tiempo de servicio de los grupos de docentes desempeñan un rol importante en la predisposición hacia el cambio y la aceptación de un esquema innovador que incrementará sus tareas cotidianas. La motivación hacia un cambio organizacional se verá fortalecida, si se crea un sistema de estímulos y reconocimientos docentes para que se destaque su nivel de profesionalismo y su autoestima, así, el profesorado se sentirá recompensado por su esfuerzo y dedicación.

Por lo tanto, para afrontar este reto de manera crítica y creativa, esta renovación parte de la sensibilización a través del desarrollo de talleres de autoestima profesional, capacitaciones sobre liderazgo participativo, temas de inclusión educativa, apertura de espacios para la recreación y confraternidad, de tal modo que contribuya en el mejoramiento académico, el clima institucional y la integración docente, a través de la formación de equipos de trabajo cooperativo.

Actitudes del estudiantado. La presencia de estudiantes con habilidades diferentes en el aula de clase ha creado un desconcierto entre compañeras y compañeros pues, por incomprensión e influencias culturales, muestran actitudes de rechazo y agresividad ante quienes son diferentes, provocando su aislamiento en medio del salón de clase. Sin embargo, se ha constatado que esas actitudes suelen suceder por sus experiencias donde 
están viviendo, de manera que, si perciben el rechazo o indiferencia ante los procesos de inclusión por parte de sus padres, madres, acudientes, familiares o amigos de la familia, entonces, es probable que asuman las mismas actitudes en la escuela.

Por consiguiente, se propone la orientación docente y la formación del alumnado dentro de un entorno donde el clima escolar sea positivo, promueva la comunicación asertiva, la disposición de materiales didácticos, la estética y la organización de ambientes físicos integrados. En tal sentido, la contextualización de las siete aulas como grandes sectores de juego-trabajo (psicomotricidad, mini cocina, expresión plástica, observación de la naturaleza, construcción, teatro, música, investigación y tecnología), en las cuales participarán de manera rotativa, será de vital importancia para el uso de las técnicas de intervención y el trabajo cooperativo que incidirá en el desarrollo de un buen clima de aula frente a las características propias de la niñez con habilidades diferentes.

Madres y padres de familia. Si bien es cierto que madres y padres conocen que la institución atiende los procesos inclusivos, también se sabe que la misma institución no les ha orientado ni involucrado por la falta de organización y por la ausencia de la gestión. $\mathrm{Al}$ respecto, se debe organizar una base de datos, elaborar un plan de acciones estratégicas, formar equipos de trabajo cooperativo y realizar talleres de sensibilización o capacitación, e involucrarles en la preparación de actividades pedagógicas, como, por ejemplo, en la definición y la aplicación de técnicas de intervención. De esa manera, mientras apoyan al personal docente, estarán en la capacidad de orientar a sus hijas e hijos acerca de la tolerancia, el respeto, la comprensión, el trato adecuado a la niñez con habilidades diferentes y a todas las personas en general.

Auxiliares y servicios psicólogos. El personal auxiliar está sujeto a las orientaciones de cada docente del aula. Resulta fundamental que este personal sea capacitado en el manejo de las estrategias, junto al personal docente y que se cuente con un recurso profesional de psicología adicional para que puedan atender a la diversidad de estudiantes, madres y padres de familias de forma permanentemente. En suma, se propone el trabajo cooperativo entre el personal docente, el personal auxiliar y los servicios psicólogos para que puedan estimular las potencialidades del alumnado, de modo que, desde sus funciones, compartan objetivos comunes debidamente articulados para el fortalecimiento de la educación inclusiva en la institución.

El modelo contextualizado para la inclusión de estudiantes con habilidades diferentes diseñado ofrece una propuesta útil, pertinente y poderosa que contribuye a la mejora del servicio educativo inclusivo en la educación inicial. El trabajo cooperativo es una estrategia promotora de valores humanos, culturales y sociales que se integra en el mismo objetivo de la comunidad educativa, la unidad de acción, la coherencia en las actividades, las relaciones humanas y el clima institucional favorable para garantizar la gestión del aprendizaje.

Validar el impacto de la propuesta en la práctica con el propósito de comprobar su efectividad en el tratamiento y desarrollo de la inclusión de estudiantes con habilidades diferentes, la eficiencia del personal docente y la comunidad pedagógica, en general, al dirigir el proceso educativo, permitirá continuar con este tipo de estudios; pero abordando otras aristas del tema y teniendo en cuenta su incidencia en la formación integral para la vida.

\section{REFERENCIAS}

Abu-Heran, N., Abukhayran, A., Domingo, J. y Pérez-García, M. (2014). Perceptions and Expectations of Palestinian Teachers towards Inclusive Education in Bethlehem District. Electronic Journal of Research in Educational and Psychology, 12(2), 461-482. doi: 10.14204/ejrep.33.14010

Alianza por la Calidad de la Educación (2010a). Módulo I: Modelo de gestión educativa estratégica. México: Secretaría de Educación Pública. Recuperado de http://formacion.sigeyucatan.gob.mx/formacion/materiales/5/d3/p3/3. \%20EL\%20MODELO\%20DE\%20GESTION\%20EDUCATIVA\%20ESTRATEGICA.pdf

Alianza por la Calidad de la Educación. (2010b). Módulo VI: Guía para facilitar la inclusión de alumnos y alumnas con discapacidad en escuelas que participan en el PEC. México: Secretaría de Educación Pública. 
Bernaldo, M. (2012). Psicomotricidad. Guía de evaluación e intervención. Madrid: Pirámide. Recuperado de https:// www.uv.mx/rmipe/files/2017/02/Psicomotricidad-guia-de-evaluacion-e-intervencion.pdf

Bristol, L. (2015). Leading-for-inclusion: Transforming action through teacher talk. International Journal of Inclusive Education, 19(8), 802-820. doi: https://doi.org/10.1080/13603116.2014.971078

Castellanos, D., Castellanos, B., Llivina, M. J., Silverio, M., Reinoso, C. y García, C. (2007). Aprender y enseñar en la escuela. La Habana: Editorial Pueblo y Educación.

Cejas, A. (2009). Gestión educativa. Revista Integra Educativa, 2(3), 215-231. Recuperado de http:// www.scielo.org.bo/scielo.php?script=sci_arttext\&pid=S1997-40432009000300010

Choza, T. (2010) Percepciones de docentes de colegios privados regulares de Lima sobre el programa de inclusión escolar (Tesis de licenciatura). Pontificia Universidad Católica del Perú, Lima, Perú.

Cruz, R. (2014). Integración e inclusión educativa vs. integración e inclusión excluyente: Un desafío educativo. Revista Mexicana De Investigación Educativa, 19(63), 1285-1290. Recuperado de http://www.scielo.org.mx/ scielo.php?script $=$ sci_arttext\&pid $=$ S1405-66662014000400013

Delors, J. (1996). La educación encierra un tesoro. Paris: Ediciones Unesco.

Fernández, J. M. y Hernández, A. (2013). El liderazgo como criterio de calidad en la educación inclusiva. Estudios Sobre Educación, 24, 83-102. Recuperado de https://www.unav.edu/publicaciones/revistas/index.php/ estudios-sobre-educacion/article/view/2025

García, A. (2014). La atención al alumnado de educación especial en Europa. Revista Española de Educación Comparada, 24, 199-222. doi: https://doi.org/10.5944/reec.24.2014.13585

Gibbs, G. (2012). El análisis de datos cualitativos en la investigación cualitativa. Madrid: Morata.

González-Moreno, C. X. (2015). Formación de la función simbólica por medio del juego temático de roles sociales en niños preescolares. Revista de la Facultad de Medicina, 63(2), 235-241. doi: https://doi.org/10.15446/ revfacmed.v63n2.47983

Kholer, J. (2004). Felicidad y modificabilidad cognitiva. Redalics. Liberabit, Revista Psicológica, 10, 68-81.

Klingler, C. y Vadillo, G. (2000). Psicología cognitiva. Estrategias en la práctica docente. México: McGraw-Hill.

López, H. (2012). Detección y evaluación de necesidades educativas especiales: Funciones del psicólogo como personal implicado en la atención a la diversidad desde los servicios educativos. Psicología Educativa, 18(2), 171-179. doi: https://doi.org/10.5093/ed2012a17

Martín, D. (2012). Psicomotricidad e intervención educativa. Madrid: Ediciones Pirámide.

McAllister, K., y Hadjri, K. (2013). Inclusion and the special needs (SEN) resource base in mainstream schools: physical factors to maximise effectiveness. Support For Learning, 28(2), 57-65. doi: https:// doi.org/10.1111/1467-9604.12019

McGlynn, C., y London, T. (2013). Leadership for Inclusion: Conceptualising and Enacting Inclusion in Integrated Schools in a Troubled Society. Research Papers In Education, 28(2), 155 -175. doi: https:// doi.org/10.1080/02671522.2011.600458

Molina, M. (2010). Estrategias didácticas de sensibilización sobre el proceso de inclusivo para maestras y maestros de pre básicas del Centro Educativo Alborada (Tesis bachelor). Universidad de Cuencia, Cuenca, Ecuador. Recuperado de http://dspace.ucuenca.edu.ec/bitstream/123456789/2847/1/te4132.pdf

Moragón-Alcañiz, F. y Martínez-Bello, V. (2016). Juegos de niñas y juegos de niños: Estudio sobre la representación del juego infantil a través del dibujo. Revista Educación, 40(1), 1-17. doi: http://dx.doi.org/10.15517/ revedu.v40i1.17439

Moriña, A. (2011). Aprendizaje cooperativo para una educación inclusiva: Desarrollo del programa PAC en un aula de educación primaria. Estudios sobre educación, 21, 199-216. Recuperado de https://dadun.unav.edu/bitstream/10171/22625/2/Art\%c3\%adculo_10_Aprendizaje \%20cooperativo\%20para\%20una\%20educaci\%c3\%b3n.pdf 
Novachevska, I., Dimchevska, I., Pavlovska, S., Chichevska-Jovanova, N. y Dimitrova-Radojichikj, D. (2011). Special education-professional and scientific issues; inclusive culture in pre-school institutions. Journal of Special Education and Rehabilitation, 12(1-2), 7-16. doi: https://doi.org/10.2478/v10215-011-0001-y

Okuda, M.y Gómez-Restrepo, C. (2005). Métodos de la investigación cualitativa: Triangulación. Revista Colombiana de Psiquiatría, 34(1), 118-124. Recuperado de http://www.scielo.org.co/pdf/rcp/v34n1/v34n1a08.pdf

Opertti, R. (2005). 48 reunión de la Conferencia Internacional de Educación: Inclusión educativa. El camino del futuro. Un desafío para compartir. Ginebra: OIE Unesco. Recuperado de https://des-mza.infd.edu.ar/sitio/ upload/RenatoOpertti_InclusionEduc.pdf

Palacios, A. (2008). El modelo social de discapacidad: orígenes, caracterización y plasmación Convención Internacional sobre los Derechos de las Personas con Discapacidad. Madrid: Cermi.

Pedraja-Rejas, L., Rodríguez-Ponce, E., y Villarroel-González, C. (2012). Los estilos de liderazgo y la gestión de recursos humanos: Una reflexión acerca del impacto sobre el desempeño de los estudiantes en la educación media. Revista Chilena de Ingeniería, 20(3), 376-385. doi: https://doi.org/10.4067/S0718-33052012000300012

$\mathrm{Qu}, \mathrm{L}$. (2011). Two is better than one, but mine is better than ours: is Preschoolers' executive function during co-play. Journal of Experimental Child Psychology, 108(3), 549-566. doi: https://doi.org/10.1016/j.jecp.2010.08.010

Ramenzoni, V. C., Riley, M. A., Shockley, K., y Baker, A. A. (2012). Interpersonal and intrapersonal coordinative modes for joint and single task performance. Human Movement Science, 31(5), 1253-1267. doi: https:// doi.org/10.1016/j.humov.2011.12.004

Ruiz, E. y Estrevel, L. B. (2010). Vygotski: La escuela y la subjetividad. Pensamiento Psicológico, 8(15), 135-145. Recuperado de http://www.redalyc.org/articulo.oa?id=80115648012

Senge, P. M. (2000). La danza del cambio: Los retos de sostener el impulso en organizaciones abiertas al aprendizaje. Bogota\#: Norma.

Solano, R. R. (2010) Conocimiento sobre educación inclusiva en los docentes del nivel inicial de colegios estatales del distrito de Bellavista, Callao (Tesis de maestría). Universidad San Ignacio de Loyola, Lima, Perú. Recuperado de http://repositorio.usil.edu.pe/bitstream/123456789/1309/1/2010_Solano_Conocimiento \%20sobre\%20educaci\%C3\%B3n\%20inclusiva\%20en\%20los\%20docentes\%20del\%20nivel\%20inicial\%20de \%20colegios\%20estatales\%20del\%20distrito\%20de\%20Bellavista-\%20Callao.pdf

Torrealba, M., Soto, N. y Piña, B. (2010). 2. El docente novel y la planificación pedagógico administrativa del proceso enseñanza aprendizaje en educación primaria. Educare, 14(2), 31-51. Recuperado de http://revistas.upel.edu.ve/ index.php/educare/article/view/359/167

Tobón, S. y Jaik, A. (Coords.). (2012). Experiencias de aplicación de las competencias en la educación y el mundo organizacional. México: Redie

Unesco (2011). Manual de gestión para directores de instituciones educativas. Perú: Unesco en Perú. Recuperado de http://unesdoc.unesco.org/images/0021/002191/219162s.pdf

Van Gigch, J. P. (1987). Teoría general de sistemas. México: Editorial Trillas.

Vargas, Z. R. (2009). La investigación aplicada: Una forma de conocer las realidades con evidencia científica. Revista Educación, 33(1), 155-165. doi: https://doi.org/10.15517/revedu.v33i1.538

Vygotski, L. S. (1979). El desarrollo de los procesos psicológicos superiores. Barcelona: Crítica.

Wallon, H. (2007). La evolución psicológica del niño. Barcelona: Ares y Mares.

\section{BY-NC-ND}

